\title{
Intermittency in an interacting generalization of the geometric Brownian motion model
}

\author{
Reimer Kühn ${ }^{1}$ and Peter Neu $^{2}$ \\ ${ }^{1}$ Mathematics Department, King's College London, Strand, London WC2R 2LS, UK \\ 2 The Boston Consulting Group, An der Welle 3, D-60322, Frankfurt, Germany
}

Received 29 November 2007, in final form 8 February 2008

Published 30 July 2008

Online at stacks.iop.org/JPhysA/41/324015

\begin{abstract}
We propose a minimal interacting generalization of the geometric Brownian motion model, which turns out to be formally equivalent to a model describing the dynamics of networks of analogue neurons. For sufficiently strong interactions, such systems may have many meta-stable states. Transitions between meta-stable states are associated with macroscopic reorganizations of the system, which can be triggered by random external forcing. Such a system will exhibit intermittent dynamics within a large part of its parameter space. We propose market dynamics as a possible application of this model, in which case random external forcing would correspond to the arrival of important information. The emergence of a model of interacting prices of the type considered here can be argued to follow naturally from a general argument based on integrating out all non-price degrees of freedom from the dynamics of a hypothetical complete description of economic dependences.
\end{abstract}

PACS numbers: 02.50.-r, 05.40.-a, 89.65.Gh, 89.75.Da

\section{Introduction}

Much effort has been devoted in recent years to understanding the statistics of price changes in financial markets (for reviews, see [1-3]).

One of the early formalizations is the geometric Brownian motion model (GBM), according to which the relative change of a price $S_{i}$ of a stock $i$ performs a random walk, captured by a Langevin equation of the form

$$
\frac{1}{S_{i}} \frac{\mathrm{d} S_{i}}{\mathrm{~d} t}=\mu_{i}+\sigma_{i} \eta_{i}(t)
$$

In equation (1), $\eta_{i}(t)$ denotes a Gaussian white noise with zero mean and unit variance, $\mu_{i}$ characterizes the average (exponential) growth rate of the stock price $S_{i}$ and the volatility $\sigma_{i}$ measures the strength of the fluctuations about the average exponential growth. Growth rates $\mu_{i}$ and volatilities $\sigma_{i}$ could in principle be extracted from observations of real market data over a suitable time span using standard regression methods. Observed correlations between price 
increments of different stocks would entail $\eta_{i}(t)$ to be correlated between different stocks, with correlation coefficients $\rho_{i j}$. The parameters thus extracted from market data might then be used, e.g., as input for portfolio optimization or derivative pricing procedures. There is a caveat, though.

Although the GBM provides a reasonable first account of the statistics of price changes, it is known to be inadequate in important respects. In particular, tails in distributions of returns are underestimated, and the intermittent character of true market dynamics manifesting itself in strong volatility fluctuations is not captured at all.

The fact that real markets are in fact not well described by GBMs of the simple form (1) with time-independent parameters $\mu_{i}$ and $\sigma_{i}$ implies that parameters extracted from observed prices are not necessarily very useful or reliable in practical applications. Interestingly, there is an important exception where some of the deficiencies of the GBM can be circumvented, namely option pricing using Black-Scholes theory based on GBMs with (possibly) timedependent parameters (see, e.g., [4]). It turns out that, remarkably, unknown time-dependent growth rates of stocks disappear from the option pricing problem, leaving only the problem of estimating volatilities to be solved. This problem is frequently addressed in 'inverse form', inferring (time-dependent) the so-called implied volatilities [4] from the prices of options actually traded in the market, a problem regarded to be stable and useful enough to be used in the financial industry.

Efforts at improving upon the GBM itself have broadly followed two main directions. In one, better characterizations of the statistics of price changes have been sought, in which the Gaussian increments of the GBM are replaced by increments following other (stable) laws (e.g. $[5,6])$. In the other, the statistics of price changes is investigated by looking at the mechanisms underlying market dynamics, for instance, by formulating them, implicitly or explicitly, as the collective result of actions of agents operating in a market. Percolation models [7-9], the minority game (MG) and its ramifications [10-12], or Ising-type models of interacting agents describing herding effects (e.g. $[13,14])$ might be mentioned in this respect.

In the present contribution, we explore an approach which is in some sense intermediate between the two lines of research just mentioned. That is, we shall not at all be explicit about the possible 'mechanics' of a market as might be formalized in terms of demand and supply, of strategies of agents operating in a market, the role of limit orders, the influence of liquidity or traded volumes or the like. Rather, we shall arrive at an interacting generalization of the GBM (to be referred to as iGBM in what follows), which derives its main motivation from very general considerations concerning projected dynamics, and begin to explore its properties.

Specifically, instead of assuming that relative price changes follow a (perhaps correlated) GBM model, we assume that their evolution depends to a certain extent on the configuration of prices of all other stocks traded in a market. This leads to an interaction between prices which may be thought of as arising effectively through the collection of agents, each acting on the basis his or her own, more or less rational perception of the underlying economy and market mechanisms. In toto these interactions would constitute a collective representation of the underlying economic dependences at the level of the dynamics of prices. Within a dynamics of prices, interactions are clearly required to generate non-trivial sequential cross-correlations between prices of different assets as they are observed in real markets (for a recent study, see [15]). Conversely, considerations about projected dynamics as detailed in section 2 will tell us that interactions between prices are in fact quite generally unavoidable, within a description of market dynamics based on prices alone.

We will demonstrate that such a setting also offers simple and transparent mechanisms to produce intermittent market dynamics. Models of financial markets producing some of the so-called stylized facts characteristic of real markets have been introduced before, notably the 
percolation model $[7,8]$ mentioned before, as well as grand-canonical variants of the MG $[16,17]$, Ising-type models also including a non-trading option state [13] or autoregressive models which explicitly include a non-trivial volatility dynamics [18]. The present proposal differs from previous ones in particular by the fact that it links up rather closely with the GBM as the classical standard model of financial markets. Unlike the other models mentioned above, it is formulated entirely in terms of prices, and it deals with the dynamics of a collection of prices, rather than looking at effects produced by a collection of agents on price formation for a single traded asset. Within the present paper our main emphasis is on suggesting a robust mechanism possibly underlying volatile price dynamics that does not require fine tuning of parameters.

However, we should like to emphasize that — apart from issues of modelling the dynamics of financial markets - the present model is interesting in its own right as a simple interacting nonlinear dynamical system exhibiting intermittent dynamics.

The remainder of this paper is organized as follows. In section 2 we introduce our model of iGBMs, adding two simple and plausible hypotheses to the standard GBM model, and demonstrate that the resulting dynamical system becomes formally equivalent to a description of the dynamics of graded response neurons. This correspondence provides the heuristics which leads us to expect that simple and robust mechanisms will exist in such systems that can create a 'market dynamics' exhibiting typical intermittent features of real markets. A brief analysis of the collective properties of the system in section 3 is used to guide finding parameter settings for which intermittent market dynamics is actually expected to occur. The main outcome of this analysis is that no fine tuning of parameters is required to achieve the desired effects. Section 4 provides a numerical simulation of the proposed framework, supplemented by a simple mechanism providing external perturbations to the market dynamics, such as could be caused by incoming information. Intermittent market dynamics with fattailed distributions of log-returns as well as volatility clustering is, indeed, observed in the system and quantitatively analysed. The concluding section, section 5, provides a summary of our results and attempts to put them into a wider perspective.

\section{The model}

\subsection{Structure and motivation of terms}

In order to set up the model, it is useful first to rewrite the GBM in terms of fields $h_{i}(t)$ defined as $h_{i}(t)=\log \left[S_{i}(t) / S_{i 0}\right]$, with $S_{i 0}$ setting a natural unit for $S_{i}(t)$. In terms of the $h_{i}(t)$, equation (1) transforms (upon setting $I_{i}=\mu_{i}-\frac{1}{2} \sigma_{i}^{2}$ and using Ito's Lemma) into

$$
\frac{\mathrm{d} h_{i}}{\mathrm{~d} t}=I_{i}+\sigma_{i} \eta_{i}(t)
$$

Starting from this formulation of the GBM (1), we propose our iGBM to take the form

$$
\frac{\mathrm{d} h_{i}}{\mathrm{~d} t}=I_{i}-\kappa_{i} h_{i}+\sum_{j(\neq i)} J_{i j} g\left(h_{j}\right)+\sigma_{i} \eta_{i}(t)
$$

with $\eta_{i}(t)$ denoting Gaussian white noises as before, albeit uncorrelated to keep matters simple.

The first addition describes a reverting force that ensures the stability of the system in the long-term limit. It would ultimately prevent the fields from growing without boundsequivalently keep stock prices on the scale of their natural unit $S_{i 0}$. Such a term might reflect the influence of fundamentalist traders on global market dynamics. The second contribution describes an interaction among prices that is motivated by underlying economic dependences among the companies whose stocks are being traded in the market. Such a term could describe 
the co-evolution of stock prices of mutually dependent firms. In equation (3), we take $g(h)$ to be some nonlinear function of the fields, describing details of the feedback mechanism that might be at work. A possible choice is a sigmoid function such as a hyperbolic tangent or an error function which varies most sensitively for prices in the vicinity of their natural value. Another possible choice is a suitable three-state function representing 'buy', 'hold' or 'sell' recommendations that exist for a given stock.

Before proceeding, it is perhaps worth commenting on the absence of the Martingale property of (3), given the prominent role attached to it in mathematical finance, and in particular for establishing no-arbitrage conditions generally held to be a fundamental property of market dynamics. The point, of course, is that it is not the bare price process (3) that is required to have the Martingale property-even the financial standard model, non-interacting GBMs, does not share this property as soon as prices exhibit a non-zero drift-but rather that a risk-adjusted process can be constructed in which (generally time-dependent) drift terms of the original process are replaced by the (possibly time-dependent) risk-free interest rate. This requires that an associated Girsanov transformation between probability measures be well defined. The details are fairly technical. A sufficient condition is the so-called Novikov condition [19, 20] which, in essence, requires that integrals over a suitably defined risk-premium exist. Suffice it to mention here that this condition is easily satisfied for processes with constant volatilities $\sigma_{i}$, as long as the processes themselves remain bounded in any bounded interval. If anything, the mean reverting terms appearing in (3) help to satisfy the latter condition. We refer to the appendix for a brief account of the formal reasoning.

\subsection{A justification from projected dynamics}

The following line of reasoning based on general consideration about projected dynamics could be advanced to justify a model of interacting price degrees of freedom. It runs like this: suppose one were to start out from a hypothetical complete theory of market dynamics, formulated in terms of demand and supply, of strategies of agents operating in a market, the role of limit orders, exchange rates, the influence of liquidity, traded volumes and so on, and suppose one were to integrate out all but the price degrees of freedom from this underlying full theory, using, e.g., Mori-Zwanzig projection techniques [21], thereby obtaining a reduced theory involving price degrees of freedom only. Such a reduced theory would then generally exhibit the following two features: (i) it would contain effective interactions between prices and (ii) it would in general be non-Markovian. Our proposal retains property (i), but not (ii). Within the framework just discussed our model would in its present form thus only constitute a Markovian approximation to a full reduced theory. We note further that higher order interactions may normally also be expected to appear in a projected dynamics. Restricting the present version to pair interactions follows widespread intuition and practice that these would create the dominant effects. Clearly, neither restriction is a matter of principle. For example, the Markovian nature of the model suggested above could easily be relaxed by including memory terms into the dynamical rules (as, indeed, they appear in the Minority game to mention a popular example [10-12]). We adopt our simplifications here mainly to keep matters simple. In this sense our model might be regarded as a minimal interacting generalization of the GBM.

Prior to actually specifying the couplings and the (nonlinear) functions $g$, our reformulation of the GBM is actually claiming nothing but the fact that (i) prices do effectively interact, this interaction being a representation of the true underlying economic dependences and that (ii) there may be reverting forces, however weak, which ensure the stability of the market in the long-time limit. 


\subsection{Correspondences}

Remarkably, the present interacting generalization of the GBM, if formulated at the level of the fields, is formally identical to a description of the stochastic evolution of a system of graded response neurons [22], with $h_{i}(t)$ playing the role of post-synaptic potentials, $J_{i j}$ denoting synaptic couplings, $g(h)$ specifying the neural input output relation and $\kappa_{i}$ designating trans-membrane conductances leading to leakage currents across membranes, while $I_{i}$ specify external input currents.

The importance of this correspondence is in the fact that it immediately suggests heuristics concerning global properties of such systems and ways of analysing them, which have been successful in the context of spin glasses and neural networks before. Indeed, a lot is known about systems of this type [22-26], which can be brought to bear on the analysis of the corresponding market model. In the absence of noise, the dynamics is governed by a Lyapunov function provided that the couplings are symmetric and that the function $g(h)$ describing the feedback mechanism is monotonically increasing [22]. Fairly general methods exist to elucidate the macroscopic properties of its attractors in that case; e.g., for the Hopfield model, phase diagrams exhibiting a 'paramagnetic' phase at large $\kappa$ and, depending on the degree of frustration in the couplings, 'glassy' as well as 'ferromagnetic' phases at small $\kappa$ were computed $[23,24]$. Moreover, in glassy phases at small $\kappa$ (and small $I_{i}$ ) the system has exponentially many attractors [26], which are expected to remain meta-stable in the weak noise limit ${ }^{3}$. For fully asymmetric Gaussian random couplings of zero mean, the noiseless dynamics (at $I_{i}=0$ ) is known to be chaotic at sufficiently small $\kappa$, whereas it is regular at large $\kappa[25]$.

Another interesting correspondence with a quite different interpretation of the equation of motion-and its own rich spectrum of collective behaviour-follows from the formal similarity of the noiseless limit of equation (3) with the equation describing the update of strategy scores in a two-strategy batch version of the minority game [10], in which case the couplings are anti-Hebbian, and $I_{i}$ are not independent of the couplings, the common choice for the nonlinear function $g$ being $g(h)=\operatorname{sign}(h)$ in that case.

\subsection{Choices}

Given the numerous possibilities that could be followed, some specialization is called for at this point. In what follows, we look at a system with Gaussian random couplings of the form $J_{i j}=\frac{J_{0}}{N}+\frac{J x_{i j}}{\sqrt{N}}$, where $x_{i j} \sim \mathcal{N}(0,1)$ with $\overline{x_{i j} x_{j i}}=\alpha$. That is, for $\alpha=1$ one has symmetric couplings, whereas for $\alpha=0, J_{i j}$ and $J_{j i}$ are uncorrelated. We emphasize that this choice is not singled out by considerations specific to market dynamics. In fact, we have no reason to expect that such a generic model will reproduce the salient features of true market dynamics faithfully in all detail. On the positive side, one might note that Gaussian distributions constitute maximally unbiased distributions subject to the constraints of given mean and variance; in this sense the choice above is one of the most sensible ones to make, prior to any empirical study that would actually attempt to extract effective couplings from real market data. Moreover, and most importantly, we find that our choice offers enough possibilities to elucidate our claim that iGBMs provide simple and transparent way to describe phenomena such as intermittent market dynamics, and qualitatively even reasonably well.

3 It appears that low-dimensional models of market dynamics exhibiting meta-stable states have appeared in the literature before, e.g., [27]. 


\section{Collective properties}

To characterize the system macroscopically, one may start from an assumption that its weak noise limit can be described in terms of small fluctuations about stationary points (if they exist) of the noiseless dynamics, which must solve

$$
h_{i}=\kappa_{i}^{-1}\left[I_{i}+\sum_{j} J_{i j} g\left(h_{j}\right)\right] .
$$

Macroscopic properties of solutions of equation (4) are obtained via a cavity-type analysis first proposed in [24] for this type of system. Defining $v_{i}=g\left(h_{i}\right)$, magnetization $m=N^{-1} \sum_{i} v_{i}$, a spin-glass-type order parameter $q=N^{-1} \sum_{i} v_{i}^{2}$ and a susceptibility $C=N^{-1} \sum_{i} g^{\prime}\left(h_{i}\right)$, one obtains the following set of self-consistency equations describing fixed points of the noiseless dynamics:

$$
m=\langle\langle\hat{v}\rangle\rangle, \quad C=\frac{1}{J \sqrt{q}}\langle\langle z \hat{v}\rangle\rangle, \quad q=\left\langle\left\langle\hat{v}^{2}\right\rangle\right\rangle,
$$

in which $\langle\langle\cdot\rangle\rangle$ denotes an average over the $\mathcal{N}(0,1)$ Gaussian $z$ and $I_{i}$ - and $\kappa_{i}$ - distributions, and $\hat{v}=\hat{v}(I, z, \kappa)$ solves $\hat{v}=g\left(\kappa^{-1}\left[J_{0} m+J \sqrt{q} z+\alpha J^{2} C \hat{v}+I\right]\right)$. In the case of multiple solutions, a Maxwell construction is used to choose the one corresponding to the proper stable solution $[23,24]$. For symmetric couplings, $\alpha=1$, equations (5) correspond to a replica-symmetric description of the system.

For the sake of simplicity, we specialize to $\kappa_{i}=\kappa$ in what follows. The structure of the phase diagram obtained from (5) is then similar to that of the SK model [28] with $\kappa$ playing the role of temperature, exhibiting an ergodic phase at large $\kappa$ (which is paramagnetic with $m=0$, if $I_{i} \equiv 0$ ) and glassy (ferromagnetic) phases at small $\kappa$ and small (large) $J_{0}$. At $0<\alpha<1$ the validity of the assumption of having fixed points is no longer guaranteed, and chaotic solutions are observed in the noiseless dynamics at sufficiently small $\alpha$. Indeed, as $\alpha \rightarrow 0$, the instability of the ergodic phase (for $I_{i} \equiv 0$ at $\kappa_{c}=J$ ) marks a transition to chaotic dynamics, as already observed in [25].

The main point is that, wherever equations (4) have a multiplicity of (glassy) solutions, i.e. in a large part of the parameter space at small $\kappa$ and small $J_{0}$, according to equations (5), there is a simple mechanism to produce intermittency in the market dynamics. It is related to the observation that, in a system with many meta-stable states (corresponding to different overall market situations), the dynamics associated with fluctuations within a state is very different from that of transitions between different meta-stable states. The latter are often associated with major restructuring of the system and accompanied by volatility bursts of the market dynamics. Transitions can be spontaneous or induced by perturbations of the market.

\section{Simulations}

In large systems, spontaneous transitions are rare. We have looked at a simple mechanism of inducing transitions which could be interpreted as modelling the effect of incoming information. It consists in occasionally resetting the 'indicator variables' $v_{i}(t)=g\left(h_{i}(t)\right)$, in terms of which the prices of the various stocks interact, to values other than determined by equations (3); specifically we implemented it by maintaining a list of randomly chosen stocks whose indicator variables are clamped at their current negative values: $v_{i}(t) \rightarrow \bar{v}_{i}=-v_{i}\left(t_{i}\right)$ for $t>t_{i}$, with $t_{i}$ denoting the time at which stock $i$ was selected to be clamped as described; with a certain probability per unit time, a random selection among the elements of this list is made to be replaced by a (randomly chosen) other stock. This procedure mimics in a 

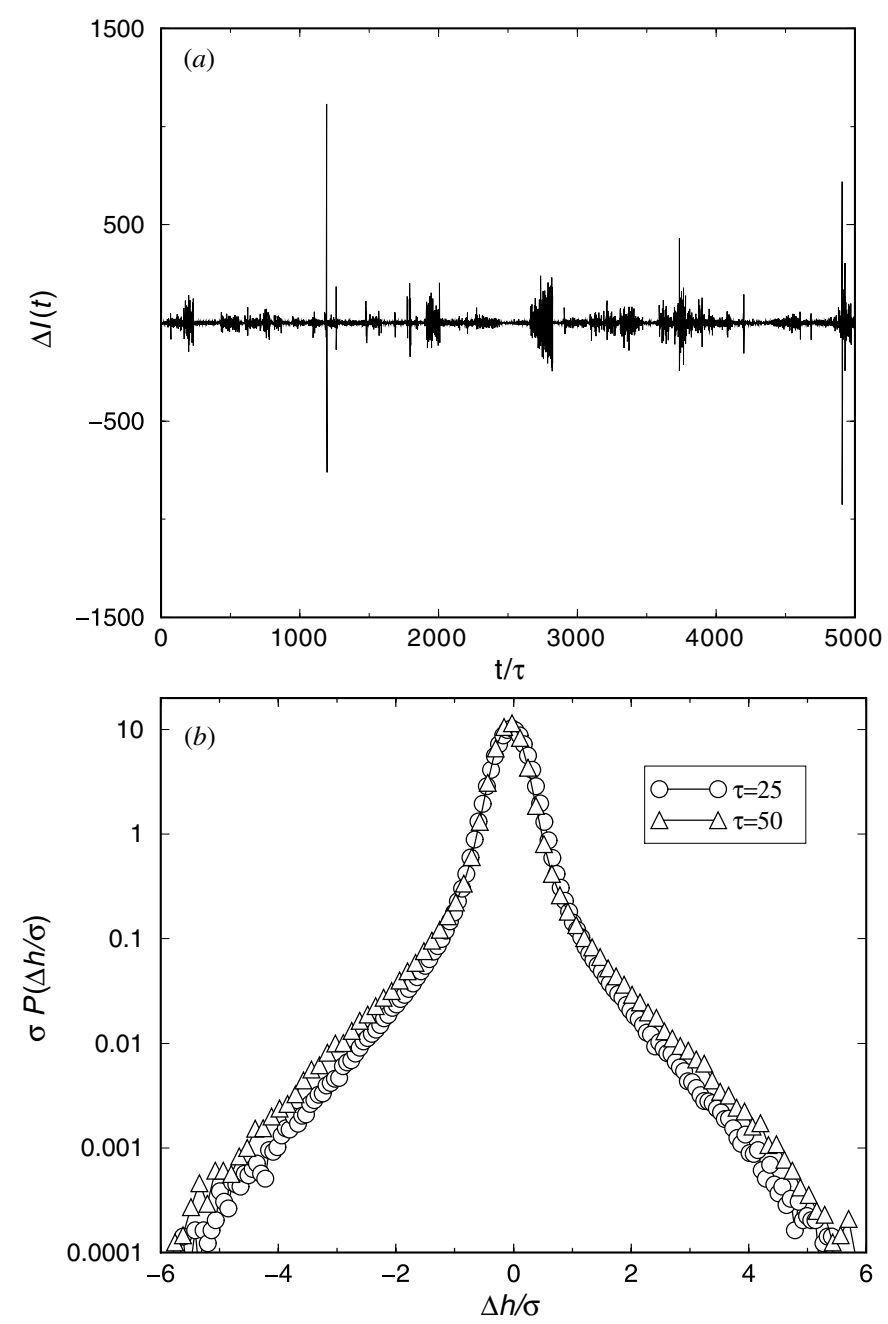

Figure 1. (a) Change of index $\Delta I$ over a time increment $\tau=25$ as a function of time for 50 interacting stocks. (b) Scaled distributions of log-returns $\sigma P(\Delta h / \sigma)$ over the index for $\tau=25$ and $\tau=50$.

generic fashion effects of unexpected information, e.g., about a company's performance on the occasion of its quarterly reporting, or a response to major analysts' reports.

The system exhibits intermittent market dynamics for a broad range of parameters, with fast decay of correlations of prices, but relatively slow decay of the volatility correlations. Here we report simulation results for a system of $N=50$ stocks with $g(h)=\tanh (h)$-feedback, system parameters $J_{0}=0, J=1, \kappa=0.4, \alpha=1 / \sqrt{2}, \mu_{i}=0$ and $\sigma_{i}^{2}$ of the form $0.05 r_{i}$ with $r_{i}$ uniform in [0.5, 1.5]. The number of stocks clamped at any time is 3 , with a random replacement in the list of clamped elements on average after $\Delta t=1000$.

Figure $1(a)$ shows the change of the index $I=\frac{1}{N} \sum_{i} S_{i}$ over a time increment $\tau=25$ as a function of time, exhibiting clear signs of intermittent behaviour. Distributions of log-returns $\left\{\Delta h_{i}=\log \left[S_{i}(t+\tau) / S_{i}(t)\right]\right\}$ shown in panel 2, both for $\tau=25$ and $\tau=50$, exhibit fat tails. Distributions at different $\tau$ scale fairly well, when normalized in terms of their variances $\sigma$. For intermediate values of normalized log-returns the distributions can be fitted to power laws 

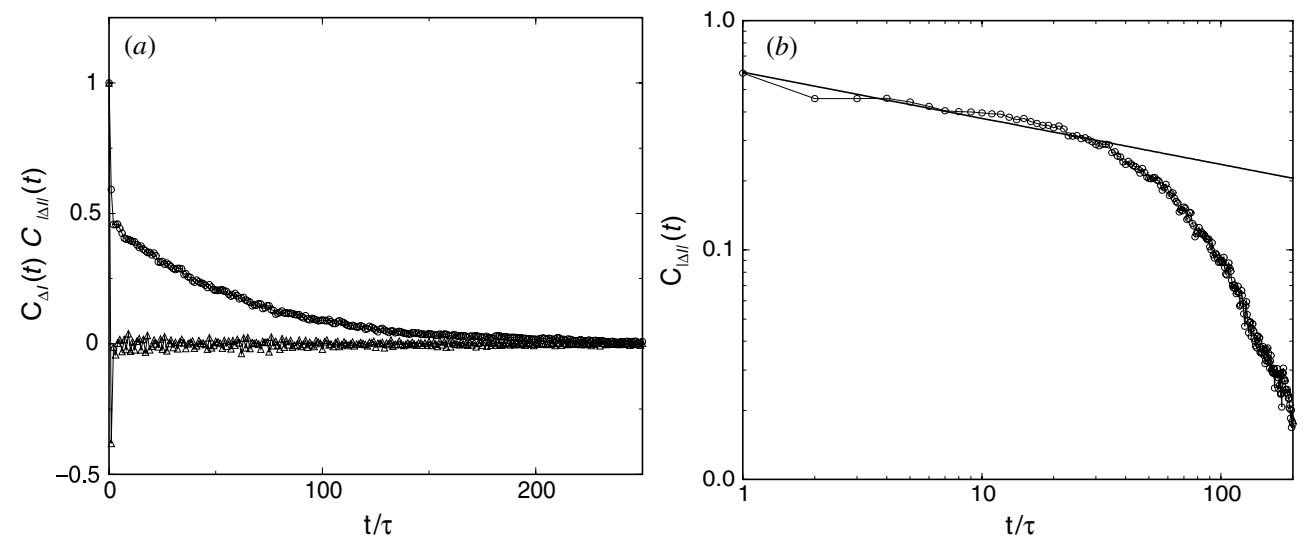

Figure 2. (a) Correlation functions of index changes (lower curve) and absolute values of index changes (upper curves) for $\tau=25$. (b) Fit to a power law decay for $t<1250(t / \tau<50)$ as described in the main text.

$P \sim \Delta h^{-(1+\mu)}$ with $\mu \simeq 2.2 \pm 0.04$ for $\tau=25$ ( $\mu \simeq 1.6 \pm 0.04$ for $\left.\tau=50\right)$, though the range covered is barely a decade, $0.4<\Delta h / \sigma<4$. Real markets typically have $\mu \simeq 3$ [29]. Figure 2(a) demonstrates that index changes $\Delta I(t)$ are anti-correlated over a small interval at short times, whereas there are long-range correlations of the 'volatilities' $|\Delta I(t)|$; as shown in figure $2(b)$, the correlation decay of the volatilities, after an initial sharp drop, is reasonably well described by a power law of the form $\sim t^{-x}$ up to $t \simeq 1250$, with $x \simeq 0.22 \pm 0.02$ for $\tau=25(x \simeq 0.27 \pm 0.02$ for $\tau=50)$. For real markets values of $x \in[0.3,0.6]$ are reported $[1,2,29]$. The crossover to exponential decay at later times (with decay time $\tau_{d} \simeq 1500$ ) is a consequence of the timescale set by the rate of external disturbances.

\section{Summary and discussion}

To summarize, we have introduced a minimal interacting generalization of the GBM and shown that it gives rise to volatile market dynamics with fat tails in distributions of log-returns and long-range correlations of volatilities. A description such as chosen here may be thought of as arising by integrating out all but the price degrees of freedom from the hypothetical underlying full theory. In such a reduced theory, interactions between prices and memory effects would normally appear, and our description would in that sense constitute a Markovian approximation to the reduced theory. Analytical studies also including non-Markovian variants of the present set-up are currently under way [31].

It is worth noting that the use of reduced theories is not uncommon in physics. The, perhaps, most prominent example is the effective electron-electron interaction obtained from an underlying theory involving electron-phonon interactions [32], a simplified version of which is then used as a cornerstone of the BCS theory of super-conductivity [33]. A similar, but less well-known example is the effective interaction between two-level systems in glasses, once more mediated by phonons, simplified versions of which have been used, e.g., to rationalize the observed universality of glassy low-temperature physics and specifically to describe lowtemperature relaxation in amorphous systems $[34,35]$. In both examples, the simplifications made in the reduced theories include the neglect of higher order interactions as well as approximations of a nature analogous to the Markovian approximation in our case. In the first example, the underlying full theory is reasonably well known. In the second example, as in 
our case, it is not, rendering theories of interacting two-level systems phenomenological in the same sense as the underlying phenomenological theories of two-level systems in glasses [36].

In many of the models suggested to reproduce the main stylized facts of market dynamics, model parameters must be tuned to create critical or near-critical conditions in these systems. For example, in the Cont-Bouchaud percolation model [7, 8], the cluster structure of trading agents must follow a statistics as obtained sufficiently close to the percolation threshold, and trading activity bust be low. A similar constraint applies, e.g., to the Ising-type model [14] in which, apart from the low trading activity constraint, the 'thermal' formation of cluster structure requires temperatures to be near critical. In a similar vein, volatile market dynamics in the grand-canonical MG $[11,12,16,17]$ requires the system to be close enough to criticality, a requirement known to become more stringent as the system size increases, and intermittent market dynamics in that system has for this reason been looked upon as a finite-size effect $[11,12,16,17]$.

Models invoking heuristics of self-organized critical behaviour [30] of market dynamics appear to constitute an exception to such fine-tuning requirement, although there is usually the requirement of a clear separation of timescales between fast internal exchange mechanisms and slow driving by the environment. The Ising-type model introduced by Iori [13] is fairly involved, and we have not been able to identify the key ingredient that makes the model critical, and in which sense, nor whether that mechanism is self-organized.

In the present model intermittent market dynamics does not require fine tuning of parameters, but appears to be a consequence of the fact that the properties of a 'marginal' glassy phase with a large number of meta-stable states (which the model would have in a large part of parameter space, if its couplings were fully symmetric) appear to survive the introduction of moderate asymmetries.

Observed effective exponents describing the distribution of log-returns and the decay of volatility correlations in the present model appear to be weakly parameter dependent, and are for the parameter settings adopted in the present paper still different from those of real markets, though not entirely off the mark (no tuning has so far been attempted). This is not unanticipated, as the present generic set-up still contains a number of features which are not entirely realistic, such as complete connectivity or uncorrelated couplings to name but two.

In the standard GBM, the model parameters $\mu_{i}$ and $\sigma_{i}$ can be directly linked to growth rates and volatilities of stock-price evolution, although the problem of reliably estimating these from observations over some finite time window is likely to be difficult if they are time dependent. It has to be pointed out that the analogous task of linking parameters of the iGBM to observable quantities is much harder and entirely non-trivial. This is due to the very existence of interactions, by which the observed growth rates and volatilities attain the status of interaction-renormalized quantities that can no longer be identified with the bare idiosyncratic growth rates and volatilities of the interacting model. Analogous issues arise in the context of credit risks, where a tentative solution involving a considerable refinement of conventional rating procedures was suggested [37]. Due to many difference in the details, a solution of that kind is infeasible in the present case, however. The additional information necessary to obtain plausible estimates for the statistics of growth rates, volatilities and interactions can only come from detailed studies of complete sets of cross-correlation functions of financial time series. However, meaningful comparisons of the quality of fits of historical data to interacting models or to non-interacting models with correlated noises should be possible and eventually allow model selection.

In this context, we believe it is worth stressing the fact that our model manifestly maintains its close links to the world of classical continuous-time finance (on this see in particular the notes in the appendix), a feature which sets it quite apart from most of the agent-based 
models discussed in the literature. In particular, it appears that issues such as derivative pricing and risk-neutral valuation are for the present model still within reach of known tools of Mathematical Finance [19, 20].

In the present study, perturbations due to unexpected incoming information were responsible for occasionally triggering phases of volatile market behaviour. Another mechanism to produce intermittent market dynamics in the iGBM we have also briefly looked at consists in assuming that the noise in (3) has a component that is slow and the same for all risk elements traded in the market. It could describe the influence of the overall economy and could with good reason be regarded as part of the internal market dynamics rather than a perturbation. In this set-up, one could take $\eta_{i}(t)=\sqrt{\rho} Y(t)+\sqrt{1-\rho} \epsilon_{i}(t)$, with $\epsilon_{i}(t)$ being independent variance-1 Gaussian white noises, and the common component $Y(t)$, e.g., a slow variance-1 Ornstein Uhlenbeck process; the parameter $0 \leqslant \rho \leqslant 1$ specifies the relative importance of the common component. Noise models of this form are actually recommended for models of financial risk within the Basel-II accord [38]. Including deterministic components in $\eta_{i}(t)$, which could, e.g., describe seasonal effects, would produce similar effects. This alternative mechanism also produced volatile market dynamics, with fast-decaying price correlations, and slowly decaying volatility correlations, but fat tails in log-returns appeared to be much less pronounced. It is suggested that the crucial difference between these noise models is that one contains jump processes, whereas the other does not. It appears reasonable to assume that in realistic markets both mechanisms-i.e., disturbances due to the arrival of unexpected information and time-dependent forces influencing the economy either as a whole or entire sectors of the economy in comparable ways-would be present to some degree.

Finally, it is perhaps worth emphasizing once more that-apart from issues of modelling the dynamics of financial markets - the present model is interesting in its own right as a simple interacting nonlinear dynamical system exhibiting intermittent dynamics.

\section{Acknowledgments}

We would like to dedicate this paper to David Sherrington on the occasion of his 65 th birthday, in recognition of his numerous contributions to the statistical mechanics of complex system as well as his prominent role in helping to establish regular and fruitful scientific contacts to colleagues in Europe, by instigating and organizing several European networks, and-on a more personal note-for help and numerous illuminating discussions throughout the years. Helpful discussions with A C C Coolen, T Galla, M Kutsia and A Løkka are gratefully acknowledged.

\section{Appendix. Arbitrage and completeness issues}

In this appendix, we briefly address the arbitrage and completeness issues of the market defined by (3). In order to make contact with the relevant theory established in continuous finance, it is useful to rewrite (3) as a set of proper stochastic differential equations to avoid (ill defined) Langevin equations,

$$
\mathrm{d} h_{i}=\left[I_{i}-\kappa_{i} h_{i}+\sum_{j(\neq i)} J_{i j} g\left(h_{j}\right)\right] \mathrm{d} t+\sigma_{i} \mathrm{~d} \tilde{W}_{i}(t),
$$

in which $\tilde{W}_{i}$ are Wiener processes. Reverting to the original price variables, one gets (using Ito's lemma)

$$
\mathrm{d} S_{i}=S_{i} \alpha_{i}(t) \mathrm{d} t+S_{i} \sigma_{i} \mathrm{~d} \tilde{W}_{i}(t)
$$


with a drift $\alpha_{i}(t)$ which is time dependent via the time dependence of the assets traded in the market, i.e., setting $\boldsymbol{S}(t)=\left\{S_{i}(t)\right\}$ we have

$$
\alpha_{i}(t)=\alpha_{i}(\boldsymbol{S}(t))=\mu_{i}-\kappa_{i} \ln \left(S_{i}(t) / S_{i 0}\right)+\sum_{j(\neq i)} J_{i j} g\left(\ln \left(S_{j}(t) / S_{j 0}\right)\right) .
$$

This is, in fact, a multidimensional version of market models extensively discussed in mathematical finance literature (e.g. [19, 20]), simplified by the facts that (i) the volatility matrix is diagonal, $\sigma_{i j}=\sigma_{i} \delta_{i j}$, and that (ii) the volatilities $\sigma_{i}$ are time independent. This model is known to be free of arbitrage, as a Girsanov transformation described by Girsanov kernels $\varphi_{i}(t)$, chosen to satisfy

$$
\sigma_{i} \varphi_{i}(t)=r(t)-\alpha_{i}(\boldsymbol{S}(t)),
$$

with $r(t)$ the risk-free interest rate, would allow to transform (A.2) into a stochastic process driven by Wiener processes $W_{i}$, and drift term given by the risk-free interest rate,

$$
\mathrm{d} S_{i}=S_{i} r(t) \mathrm{d} t+S_{i} \sigma_{i} \mathrm{~d} W_{i}(t),
$$

thereby excluding arbitrage ([20], chapter 11). In formal terms, the Girsanov transformation affects a transformation between $\left\{\tilde{W}_{i}\right\}$ path probabilities and $\left\{W_{i}\right\}$ path probabilities.

The transformation is well defined, if the so-called Novikov condition

$$
\mathrm{E}\left[\exp \left(\frac{1}{2} \int_{0}^{T} \mathrm{~d} t \sum_{i} \varphi_{i}(t)^{2}\right)\right]<\infty
$$

is satisfied, in which the expectation is defined in terms of the $\left\{\tilde{W}_{i}\right\}$ path probabilities, and the risk horizon $T$ is assumed to be finite. Since in our case

$$
\varphi_{i}(t)=\frac{r(t)-\alpha_{i}(\boldsymbol{S}(t))}{\sigma_{i}}
$$

with time-independent $\sigma_{i}$, possible violations of the Novikov condition could only come from divergences of the nominator. This possibility can, however, be excluded given the nature of (A.2) and the properties of the drift terms $\alpha_{i}(\boldsymbol{S}(t))$. If anything, the mean reverting term with the only potential divergences at $S_{i} \rightarrow 0$ or $S_{i} \rightarrow \infty$ contributes to $S_{i}(t)$ staying away from these values.

Following standard reasoning [19, 20]), one would classify a market described by (3) as complete, as the number of driving Wiener processes is taken to be equal to the number of traded risky assets. As a consequence, simple contingent claims in the sense of [20] would allow unique pricing via a multidimensional generalization of the Black-Scholes equation.

The situation is more subtle in the cases where we consider perturbed versions of the dynamics, either as investigated in section 4., or of the form mentioned in section 5. According to current understanding, the absence of arbitrage is a property likely to survive the introduction of either form of random perturbation. Completeness, on the other hand, is not, as the very introduction of random perturbations entails that the number of random processes driving the dynamics would be larger than the number of traded risky assets (on this, see the 'Meta theorem' 8.3.1 in [20]). Derivative pricing would then no longer be unique.

\section{References}

[1] Bouchaud J P and Potters M 2000 Theory of Financial Risks (Cambridge: Cambridge University Press)

[2] Mantegna R N and Stanley H E 1999 An Introduction to Econophysics (Cambridge: Cambridge University Press)

[3] Feigenbaum J 2003 Financial physics Rep. Prog. Phys. 661611 
[4] Hull J C 2000 Options, Futures and Other Derivatives 4th edn (NJ: Prentice-Hall)

[5] Mandelbrot B B 1997 Fractals and Scaling in Finance (Berlin: Springer)

[6] Mantegna R N and Stanley H E 1994 Phys. Rev. Lett. 732946

[7] Cont R and Bouchaud J P 2000 Macroecon. Dyn. 4170

[8] Stauffer D and Penna T J P 1998 Physica A 256284

[9] Stauffer D and Sornette D 1999 Physica A 271496

[10] Challet D and Zhang Y C 1997 Physica A 246407 Cavagna A 1999 Phys. Rev. E 59 R3783

Cavagna A, Garrahan J P, Giardina I and Sherrington D 1999 Phys. Rev. Lett. 834429

Heimel J A F and Coolen A C C 2001 Phys. Rev. E 63056121

[11] Challet D, Marsili M and Zhang Y C 2004 Minority Games-Interacting Agents in Financial Markets (Oxford: Oxford University Press)

[12] Coolen A C C 2005 The Mathematical Theory of Minority Games-Statistical Mechanics of Interacting Agents (Oxford: Oxford University Press)

[13] Iori G 1999 Int. J. Mod. Phys. C 101149

[14] da Silva L R and Stauffer D 2001 Physica A 294235

[15] Nakamura T and Small M 2007 Physica A 38396

[16] Slanina F and Zhang Y C 2001 Physica A 289290

Jeffries P, Hart M, Hui P M and Johnson N F 2001 Eur. Phys. J. B 20493

[17] Challet D and Marsili M 2003 Phys. Rev. E 68036132

[18] Engle R F 1982 Econometrica 50987 Bollerslev T 1986 Economet. 31307

[19] Karatzas I and Shreve S E 1998 Methods of Mathematical Finance (New York: Springer) chapter 1

[20] Björk T 2004 Arbitrage Theory in Continuous Time 2nd edn (Oxford: Oxford University Press) chapters 10 and 11

[21] Zwanzig R 1961 Lectures in Theoretical Physics (Boulder) vol 3 ed W A Brittin (New York: Interscience)

[22] Hopfield J et al 1984 Proc. Natl Acad. Sci. USA 813088

[23] Kühn R and Bös S 1993 J. Phys. A: Math. Gen. 26831

[24] Shiino M and Fukai T 1992 J. Phys. A: Math. Gen. 25 L375

[25] Sompolinsky H, Crisanti A and Sommers H J 1988 Phys. Rev. Lett. 61259 Molgedey L, Schuchhardt J and Schuster H G 1992 Phys. Rev. Lett. 693717

[26] Fukai T and Shiino M 1990 Phys. Rev. A 427459 Waugh F R, Marcus C M and Westervelt R M 1990 Phys. Rev. Lett. 641986

[27] Caginalp G 1990 Appl. Math. Lett. 417

[28] Sherrington D and Kirkpatrick S 1975 Phys. Rev. Lett. 351792

[29] Gopikrishnan P, Plerou V, Amaral L A Nunes, Meyer M and Stanley H E 1999 Phys. Rev. E 605305

[30] Bak P, Scheinkman J, Chen K and Woodford M 1993 Ricerche Economichi 473 Bak P, Tang C and Wiesenfeld K 1987 Phys. Rev. Lett. 59381

[31] Galla T, Coolen A C C, Kühn R and Sherrington D work in progress Anand $\mathrm{K}$ and Kühn $\mathrm{R}$ work in progress

[32] Fröhlich H 1952 Proc. R. Soc. A 215291

[33] Bardeen J, Cooper L N and Schrieffer J R 1957 Phys. Rev. 106162

[34] Yu C C and Leggett A J 1988 Comments Cond. Mat. Phys. 14231

[35] Burin A L, Natelson D, Osheroff D D and Kagan Yu 1998 Tunneling Systems in Amorphous and Crystalline Solids ed P Esquinazi (Berlin: Springer) pp 223-315

[36] Anderson P W, Halperin B I and Varma C M 1972 Philos. Mag. 251 Phillips W A 1972 J. Low Temp. Phys. 7351

[37] Neu P and Kühn R 2004 Physica A 342639

[38] Basel Committee on Banking Supervision 2005 International Convergence of Capital Measurement and Capital Standards: A Revised Framework (Birkhauser: Basel) (http://www.bis.org) 